\title{
Ascorbic acid improves thrombotic function of platelets during living donor liver transplantation by modulating the function of the E3 ubiquitin ligases $\mathrm{Cbl}-\mathrm{b}$ and $\mathrm{c}-\mathrm{Cbl}$
}

\author{
B.B. Han, J. H. Kwon, B. S. Shin \\ Department of Anesthesiology and Pain medicine, Samsung Medical Center \\ Sungkyunkwan University School of Medicine, Seoul, Republic Korea
}

\section{Background and Goal of Study:}

This study employed thromboelastography to investigate the effect of ascorbic acid (AA) on hemostatic function during living donor liver transplantation (LDLT). In addition, we examined whether AA affects clot rigidity by modulating the function of intracellular E3 ubiquitin ligases, Cbl-b and c-Cbl, during orthotopic liver transplantation

\section{Materials and Methods:}

Twenty-one recipients undergoing LDLT were enrolled in this study. Thromboelastography was performed using blood samples drawn within 30 min after the induction and at 120-min after reperfusion. Each blood sample was divided into three groups: the control group (C group: $0.36 \mathrm{~mL}$ of blood sample), the normal saline group ( $\mathrm{N}$ group: $0.33 \mathrm{~mL}$ of blood sample + $0.03 \mathrm{~mL}$ of $0.9 \%$ normal saline), and the $\mathrm{AA}$ group (A group: $0.33 \mathrm{~mL}$ of blood sample $+0.03 \mathrm{~mL}$ of $200 \mu \mathrm{mol}$ L-AA dissolved in $0.9 \%$ normal saline). Western blot analysis was performed after platelet isolation at the corresponding times to investigate the functional changes of $c-C b l$ and $\mathrm{Cbl}-\mathrm{b}$.

\section{Results and Discussion:}

Thromboelastographic analysis revealed that AA decreased fibrinolysis and increased clot rigidity at baseline and $120 \mathrm{~min}$ after reperfusion. Western blotting revealed that Cbl-b expression was significantly increased at baseline and $120 \mathrm{~min}$ after reperfusion in the $\mathrm{A}$ group compared to the $\mathrm{C}$ or $\mathrm{N}$ groups. However, among the three groups, phosphorylation of $\mathrm{c}-\mathrm{Cbl}$ was most significantly decreased in the A group at baseline and 120 min after reperfusion.

\section{Conclusion(s):}

Our results showed that $A A$ can significantly decrease fibrinolysis and improve clot rigidity in recipients during LDLT, and the functional changes in Cbl-b and $c-C b l$ might represent the mechanism underlying these findings. Therefore, the use of AA might be considered to improve hyperfibrinolysis during LDLT

\section{References (optional):}

[1] Czubkowski P, Socha P, Pawlowska J. Current status of oxidative stress in pediatric liver transplantation. Pediatr Transplant 2010;14:169-77.

[2] Elias-Miro M, Jimenez-Castro MB, Rodes J, et al. Current knowledge on oxidative stress in hepatic ischemia/reperfusion. Free Radic Res 2013;47:555-68.

[3] Muriel P. Role of free radicals in liver diseases. Hepatol Int 2009;3:526-36.
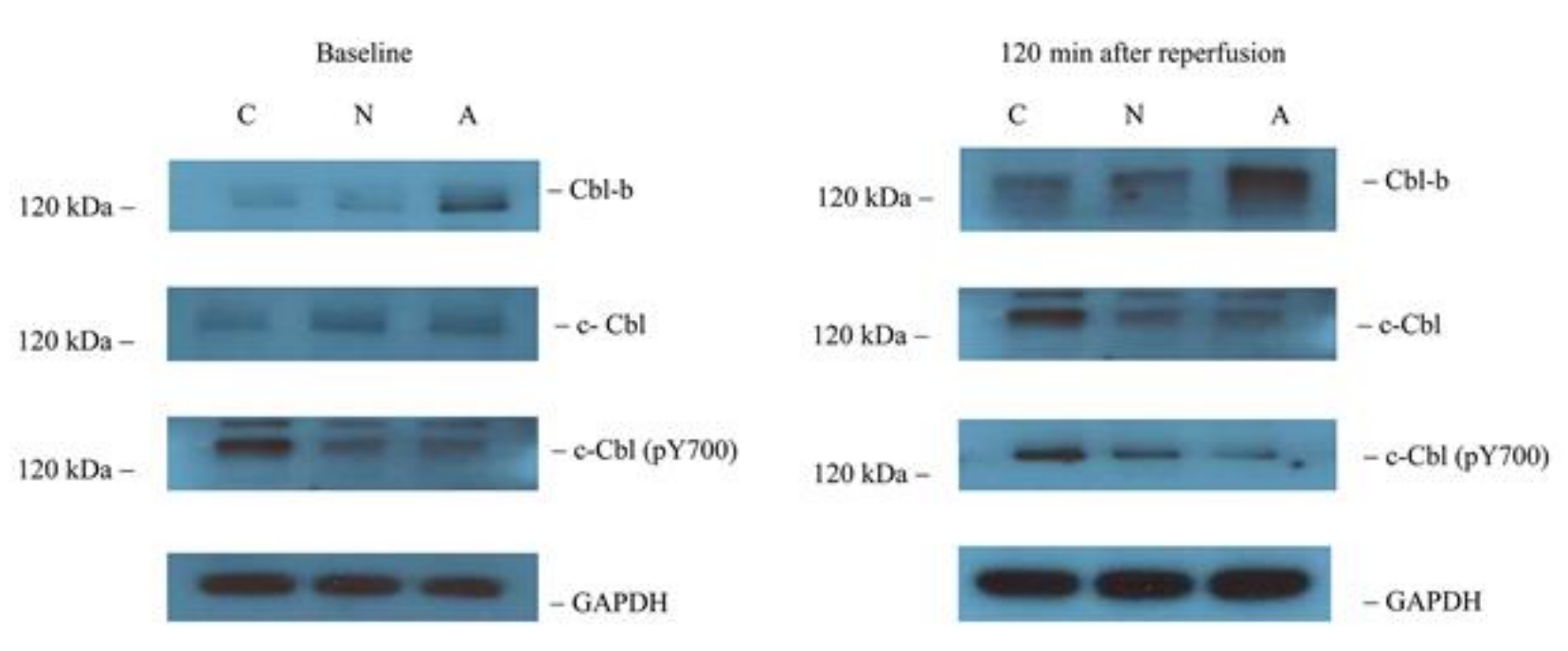

Figure 2. Western blot analysis of $\mathrm{Cbl}-\mathrm{b}, \mathrm{c}-\mathrm{Cbl}$, and phosphorylated forms of c-Cbl in $\mathrm{C}, \mathrm{N}$, and $\mathrm{A}$ groups at baseline (30 min after induction) and $120 \mathrm{~min}$ after reperfusion, respectively.

C group: control group; N group: normal saline group; and A group: AA group.

\begin{tabular}{|c|c|c|c|c|c|c|}
\hline & Baseline & & & 120 min after reperfusion & & \\
\hline Group & $\mathbf{C}$ & $\mathbf{N}$ & $\mathbf{A}$ & $\mathrm{C}$ & $\mathbf{N}$ & $\mathbf{A}$ \\
\hline $\mathrm{CT}(\mathrm{sec})$ & $905.0(745.5,1173.0)$ & $794.0(703.5,1017.0)^{\mathrm{a}, \mathrm{b}}$ & $595.0(516.0,737.0)^{\mathrm{a}, \mathrm{b}}$ & $824.4 \pm 482.7$ & $735.0 \pm 190.3$ & $593.4 \pm 151.7^{\mathrm{a}}$ \\
\hline CFT (sec) & $650.0(455.5,1007.5)$ & $542.0(378.0,815.0)^{\mathrm{a}, \mathrm{b}}$ & $407.5(282.5,751.0)^{\mathrm{a}, \mathrm{b}}$ & $688.5(488.0,1288.5)$ & $706.7 \pm 415.6^{\mathrm{a}}$ & $670.8 \pm 325.3^{\mathrm{a}}$ \\
\hline$\alpha$-angle $\left(^{\circ}\right)$ & $26.6 \pm 10.3$ & $31.3 \pm 9.3^{\mathrm{a}, \mathrm{b}}$ & $36.6 \pm 11.4^{\mathrm{a}, \mathrm{b}}$ & $30.0(19.0,33.0)$ & $32.3 \pm 11.6^{\mathrm{a}}$ & $31.6 \pm 11.0^{\mathrm{a}}$ \\
\hline A10 & $17.8 \pm 7.6 *$ & $20.7 \pm 7.6^{\mathrm{b}}$ & $25.9 \pm 8.5^{\mathrm{a}, \mathrm{b}}$ & $18.0(13.0,21.0)$ & $19.0(16.0,25.0)^{\mathrm{a}}$ & $18.0(17.0,26.0)^{\mathrm{a}}$ \\
\hline A20 & $25.3 \pm 9.8$ & $28.5 \pm 8.8^{b}$ & $34.2 \pm 10.3^{\mathrm{a}, \mathrm{b}}$ & $26.0(19.0,29.0)$ & $27.5(22.0,34.0)^{\mathrm{a}}$ & $25.5(23.5,34.5)^{\mathrm{a}}$ \\
\hline $\mathrm{MCF}(\mathrm{mm})$ & $31.6 \pm 11.9$ & $34.3 \pm 10.6^{\mathrm{a}, \mathrm{b}}$ & $38.5 \pm 10.9^{\mathrm{a}, \mathrm{b}}$ & $32.0(23.5,35.5)$ & $34.0(26.5,38.5)^{\mathrm{a}, \mathrm{b}}$ & $31.5(28.5,40.5)^{\mathrm{a}, \mathrm{b}}$ \\
\hline $\mathrm{ML}(\%$ of $\mathrm{MCF})$ & $18.5(15.0,25.0)$ & $20.3 \pm 7.2^{\mathrm{b}}$ & $14.8 \pm 6.0^{\mathrm{a}, \mathrm{b}}$ & $11.1 \pm 5.2$ & $10.7 \pm 5.5^{\mathrm{b}}$ & $6.7 \pm 1.3^{\mathrm{a}, \mathrm{b}}$ \\
\hline LI60 (\% of MCF) & $96.5(94.0,100.0)$ & $96.5(93.0,98.0)^{\mathrm{b}}$ & $98.0(96.0,100.0)^{\mathrm{a}, \mathrm{b}}$ & $100.0(99.0,100.0)$ & $100.0(100.0,100.0)^{\mathrm{b}}$ & $100.0(100.0,100.0)^{\mathrm{a}, \mathrm{b}}$ \\
\hline MAXV-t (sec) & $1018.0(898.0,1402.0)$ & $853.0(780.0,1093.0)^{\mathrm{a}, \mathrm{b}}$ & $718.0(623.5,922.0)^{\mathrm{a}, \mathrm{b}}$ & $830.5(677.5,964.0)$ & $843.0(717.0,999.0)^{\mathrm{a}}$ & $660.0(550.0,833.0)^{\mathrm{a}}$ \\
\hline MCF-t (sec) & $2325.5(2102.5,2549.0)$ & $2105.5(1945.5,2360.0)^{\mathrm{b}}$ & $2384.5(2171.5,2575.5)^{\mathrm{b}}$ & $2597.5(2483.0,2897.0)$ & $2557.5(2082.5,2779.0)^{\mathrm{a}, \mathrm{b}}$ & $2931.5(2561.5,3200.5)^{\mathrm{a}, \mathrm{b}}$ \\
\hline $\operatorname{AUC}\left(\Omega^{*} \min \right)$ & $3176.6 \pm 1191.8$ & $3502.3 \pm 974.7^{\mathrm{a}, \mathrm{b}}$ & $3893.5 \pm 1070.0^{\mathrm{a}, \mathrm{b}}$ & $3267.5(2473.5,3680.0)$ & $3369.5(2887.5,3915.5)^{\mathrm{a}}$ & $3474.5(2919.0,4082.5)^{\mathrm{a}}$ \\
\hline $\operatorname{CFR}\left({ }^{\circ}\right)$ & $31.2 \pm 9.62$ & $45.7 \pm 8.5^{\mathrm{a}}$ & $43.5 \pm 10.3^{\mathrm{a}}$ & $38.5(29.5,43.0)$ & $44.0(39.0,54.0)^{\mathrm{a}}$ & $43.0(39.0,49.0)^{\mathrm{a}}$ \\
\hline
\end{tabular}

Table1. Results of thromboelastography CT: clotting time; CFT: clot formation time; A10 and A20: amplitude 10 and 20 min after CFT, respectively; MCF: maximum clot firmness; ML: maximum lysis; LI60: lysis index at $60 \mathrm{~min}$; MAXV-t: time to maximum velocity of clot formation; MCF-t: MCF-time; AUC: area under curve from the start of measurement to 6 min; CFR: clot formation rate. a: compared with $\mathrm{C}$ group $(P<.05)$; : compared between $\mathrm{N}$ and $\mathrm{A}$ groups $(P<.05)$; $\mathrm{C}$ group: control group; $\mathrm{N}$ group: normal saline group; and $\mathrm{A}$ group: $\mathrm{AA}$ group. 